Article

\title{
Statistical Validation Verifies That Enantiomorphic States of Chiral Cells Are Determinant Dictating the Left- or Right-Handed Direction of the Hindgut Rotation in Drosophila
}

\author{
Tomoki Ishibashi $^{1}$ (D), Mikiko Inaki ${ }^{2}$ and Kenji Matsuno ${ }^{2, *(1)}$ \\ 1 Graduate School of Agriculture, Kyoto University, Kyoto 569-0073, Japan; ishibashi.tomoki.5m@kyoto-u.ac.jp \\ 2 Department of Biological Sciences, Graduate School of Science, Osaka University, Toyonaka, \\ Osaka 560-0043, Japan; minaki@bio.sci.osaka-u.ac.jp \\ * Correspondence: kmatsuno@bio.sci.osaka-u.ac.jp
}

Received: 9 November 2020; Accepted: 30 November 2020; Published: 2 December 2020

check for updates

\begin{abstract}
In the left-right (LR) asymmetric development of invertebrates, cell chirality is crucial. A leftor right-handed cell structure directs morphogenesis with corresponding LR-asymmetry. In Drosophila, cell chirality is thought to drive the LR-asymmetric development of the embryonic hindgut and other organs. This hypothesis is supported only by an apparent concordance between the LR-directionality of cell chirality and hindgut rotation and by computer simulations that connect the two events. In this article, we mathematically evaluated the causal relationship between the chirality of the hindgut epithelial cells and the LR-direction of hindgut rotation. Our logistic model, drawn from several Drosophila genotypes, significantly explained the correlation between the enantiomorphic (sinistral or dextral) state of chiral cells and the LR-directionality of hindgut rotation-even in individual live mutant embryos with stochastically determined cell chirality and randomized hindgut rotation, suggesting that the mechanism by which cell chirality forms is irrelevant to the direction of hindgut rotation. Thus, our analysis showed that cell chirality, which forms before hindgut rotation, is both sufficient and required for the subsequent rotation, validating the hypothesis that cell chirality causally defines the LR-directionality of hindgut rotation.
\end{abstract}

Keywords: cell chirality; left-right asymmetry; gut; development; Drosophila

\section{Introduction}

Left-right (LR) asymmetry is typically integrated into the basic body plan in bilaterians, in which internal organs are often LR-asymmetric in both morphology and function. The mechanisms of LR-asymmetric development are well studied in vertebrates [1-5]. In mice, for example, motile cilia located in the embryonic ventral node rotate clockwise and induce a leftward flow of extra-embryonic fluid, thereby creating the first break in LR symmetry [6]. This phenomenon, called nodal flow, subsequently induces the left-side-specific expression of genes required for LR-asymmetric organ development, such as Nodal and Lefty [6-8]. The mechanisms that break LR symmetry are evolutionarily divergent even among vertebrates; for example, LR-symmetry breaking occurs independently of nodal flow in both reptiles and chickens $[9,10]$. In invertebrates, chiral cells contribute to LR-asymmetric development (a cell is chiral if its shape cannot be superimposed onto its mirror image) [11-13]. In snails and nematodes, the chirality of blastomeres at the initial stage of cleavage determines their subsequent LR-asymmetric arrangement, which consequently directs the LR-asymmetry of the whole body $[14,15]$. 
Stereotypic LR asymmetry is evident in several organs in Drosophila, including the embryonic gut, male genitalia, and brain [16-24]. The embryonic hindgut is the first organ to show LR asymmetry in the developing Drosophila embryo [16]. The hindgut is formed as a bilaterally symmetric structure in which the anterior part curves toward the ventral side of the embryo and subsequently rotates $90^{\circ}$ counterclockwise, so that it bends toward the right [16]. The active force that drives hindgut rotation is generated by the hindgut epithelial tube $[25,26]$. Before rotation, the apical boundaries of the hindgut epithelial cells slant to the left relative to the organ's anterior-posterior axis [25,27-29]. Given the apical-basal polarity of the hindgut epithelial cells, their three-dimensional structure is chiral [27]. During hindgut rotation, the cells gradually lose chirality, becoming bilaterally symmetric [27]. Coordinated studies between computer simulations involving vertex models and image analysis of the hindgut epithelium suggest that the dissolution of cell chirality induces cell sliding, in which the epithelial cells LR-asymmetrically change their relative positions without remodeling cell junctions, and that cell sliding is coupled with the rotation of the hindgut epithelial tube in the corresponding LR direction $[25,27]$. Cell chirality contributes to LR-asymmetric development in several organs in Drosophila, suggesting that it plays general roles in the LR-asymmetric development of this species [20,30-32]. Cell chirality has been observed not only in snails, nematodes, and Drosophila, but also in Chordata, such as Larvaceans and chickens, and cell chirality is thought to be involved in these species' LR-asymmetric development as well [33,34]. In addition to cell chirality observed in vivo during development, cultured cells from diverse species, from Dictyostelium to humans, demonstrate intrinsic chirality in their structure and movement [35-39]. Thus, cell chirality is a widely observed property shared among eukaryotic cells.

Drosophila has been used as a model system to study the genetic mechanisms underlying the formation of cell chirality [27,29,32]. MyosinID (MyoID), a Myosin I family gene that is also called Myosin31DF, can induce dextral cell chirality, so that MyoID loss-of-function mutations result in sinistral cell chirality and the LR-inversion of various organs, including the embryonic hindgut, that have dextral LR-asymmetry in wild-type animals [20,25,27-30,32,40]. MyosinIC (MyoIC), a Myosin I family gene that is also called Myosin61F, can induce sinistral cell chirality, which leads to the LR-inversion of several organs with handedness when overexpressed $[17,26,40,41]$. We previously found that loss-of-function mutations of the extra macrochaetae (emc) gene, the Drosophila ortholog of $I d$, randomizes hindgut handedness [29]. Id family genes encode class V helix-loop-helix (HLH) proteins that inhibit E-box proteins-a class of basic HLH transcriptional factors, such as Daughterless (Da) in Drosophila - by forming a heterodimer, thereby regulating the balance between cell differentiation and cell proliferation [42]. We found that MyoID functions downstream of or parallel to emc in the formation of cell chirality [29]. Although the genetic mechanisms that create cell chirality are becoming clearer, a causal relationship between cell chirality and hindgut rotation is supported only by an apparent concordance between the enantiomorphic (sinistral or dextral) state of chiral cells and the direction of hindgut rotation in various mutants, along with the results of computer simulations recapitulating the rotation of the model gut tube driven by the dissolution of cell chirality [25,27]. Therefore, this hypothesized cause-and-effect relationship still needs to be validated.

In this article, we mathematically evaluated the causal relationship between the LR-directionality of cell chirality and the subsequent hindgut rotation, using a new live-imaging procedure that tests a cause-and-effect relationship between the two. Our study validated that the enantiomorphic state of chiral cells causally defines the LR-asymmetry of hindgut rotation.

\section{Materials and Methods}

\subsection{Fly Lines}

Canton-S was used as the wild-type Drosophila strain. The following mutants were used: $e m c^{A P 6}$, an amorphic allele (Bloomington \#36544) [43]; $d a^{10}$, an amorphic allele (Bloomington \#5531) [44]; and $M y o I D^{K 2}$, an amorphic allele [45]. The following UAS lines were used: UAS-emc::GFP [46]; 
UAS-da (Bloomington \#51669) [47]; UAS-MyoIC [17]; UAS-MyoID::mRFP [28]; and UAS-myr::GFP [48]. The following Gal4-driver lines were used: NP2432 (Kyoto DGRC \#104201) [49] to analyze the fixed cell-chirality index, and byn-Gal4 [17] to analyze the live cell-chirality index. Mutations on the second and third chromosomes were balanced with $C y O, P\{e n 1\} w g$ en 11 and with TM3, P\{GAL4-twi.G\}2.3, $P\{U A S-2 x E G F P\} A H 2.3, \mathrm{Sb}^{1} \mathrm{Ser}^{1}$, respectively. All genetic crosses were carried out at $25^{\circ} \mathrm{C}$ on a standard Drosophila culture medium.

\subsection{Live Imaging and Laterality Score}

The cell membrane of the hindgut epithelium was visualized by UAS-myr::GFP expression driven by byn-Gal4 using the GAL4/UAS system [50]. Drosophila embryos were dechorionated and placed on grape-juice agar plates. Embryos of the appropriate genotypes were selected at early stage 12 under a fluorescence microscope and mounted dorsal-side up on double sticky tape on slide glasses. The embryos were overlaid by oxygen-permeable Halocarbon oil 27 (Sigma-Aldrich, St. Louis, MO, USA) and a coverslip, using other coverslips of the appropriate thickness as spacers $(0.17-0.25 \mathrm{~mm})$. Live images of apical cell boundaries in the hindgut epithelium were obtained with an LSM 880 scanning laser confocal microscope before the onset of hindgut rotation. The embryo was then cultured at $25^{\circ} \mathrm{C}$ for $3 \mathrm{~h}$, at which point the rotational direction of the hindgut was evaluated, and the embryo was assigned a laterality score of 1 (counterclockwise), 0 (clockwise), or 0.5 (no rotation).

\subsection{Analysis of Cell Chirality Index}

Cell chirality was analyzed as previously described [29]. Briefly, we obtained images of the apical cell boundaries, detected by Myr::GFP signal, in the dorsal part of the embryonic hindgut just before its rotation (at stage 12) using the LSM 880 confocal microscope (Carl Zeiss, Oberkochen, Germany). Based on these images, we determined the angle $(\theta)$ between the anterior-posterior axis of the hindgut tube and each cell boundary using ImageJ Fiji v.2.0.0 [51]. To quantify the LR-asymmetric slanting of the apical cell boundaries in the hindgut epithelial cells, we calculated the cell chirality index as $\left(N_{R}-N_{L}\right) /\left(N_{R}+N_{L}\right)$, where $N_{R}$ was the number of boundaries with $0^{\circ}<\theta<90^{\circ}$ or $-180^{\circ}<\theta<-90^{\circ}$, and $N_{L}$ was the number of boundaries with $-90^{\circ}<\theta<0^{\circ}$ or $90^{\circ}<\theta<180^{\circ}$, for each embryo. The chirality index was measured in a double-blind manner, in which the person analyzing the cell boundaries did not know the genotype of the embryo or the prospective rotational direction of the hindgut.

\subsection{Statistical Analysis}

We used logistic models to test the correlation between the mean cell-chirality index and the mean laterality score in fixed embryos or between the live cell-chirality index and the laterality score in each living embryo [52]. As a null hypothesis, we used naïve models [52]. For the fixed embryos, the mean laterality score was treated as a response variable, and the mean fixed cell-chirality index was treated as an explanatory variable. For the live embryos, the laterality score of each individual embryo $(0,0.5$, or 1$)$ was treated as a response variable, and the live cell-chirality index of each was treated as an explanatory variable. All statistical analyses were performed using R software v.3.6.3 [53]. Graphs were prepared using Matplotlib v.3.0.3 in Python v.3.6.4 [54,55].

\section{Results}

3.1. A Logistic Model Significantly Explained the Correlation between the Enantiomorphic States of Chiral Cells and the LR-Directionality of Hindgut Rotation

The Drosophila hindgut is first formed as a bilaterally symmetric invagination in which the anterior part curves ventrally at early stage 12 (Figure 1A, left panel) [17]. Then, it gradually rotates counterclockwise $90^{\circ}$ as viewed from the posterior (Figure 1A, middle panels). This rotation causes the hindgut to curve toward the right at the end of stage 13 (Figure 1A, right-most panel). The hindgut 
epithelium has a typical apical-basal polarity, and the inner surface of the hindgut corresponds to the apical surface. Before the rotation of the hindgut, the chirality of the hindgut epithelial cells is detected in the shape of their apical surface, and the frequency of cell boundaries that slant to the left or right relative to the anterior-posterior axis of the hindgut tube deviates according to the genetic conditions [29]. For example, wild-type embryos had more left- than right-slanting cell boundaries (Figure 1B, left panel). However, there was no LR bias in the angle of cell boundaries when hindgut rotation was complete (Figure 1B, right panel). We quantitatively analyzed cell chirality using a previously formulated chirality index [29]. The apical cell boundaries were visualized, and two-dimensional coordinates corresponding to the anterior-posterior and left-right axes were placed on a vertex as the origin (Figure 1C, left panel). The cell boundaries in the first and third quadrants (shown in orange) were defined as Right (orange lines), while those in the second and fourth quadrants (shown in turquoise) were defined as Left (turquoise lines) (Figure 1C, left and middle panels). The number of Right and Left cell boundaries was represented by N-Right $\left(N_{R}\right)$ and N-Left $\left(N_{L}\right)$, respectively (Figure 1C, right panel). The mean cell-chirality index was calculated from the values obtained for all embryos of the same genotype.
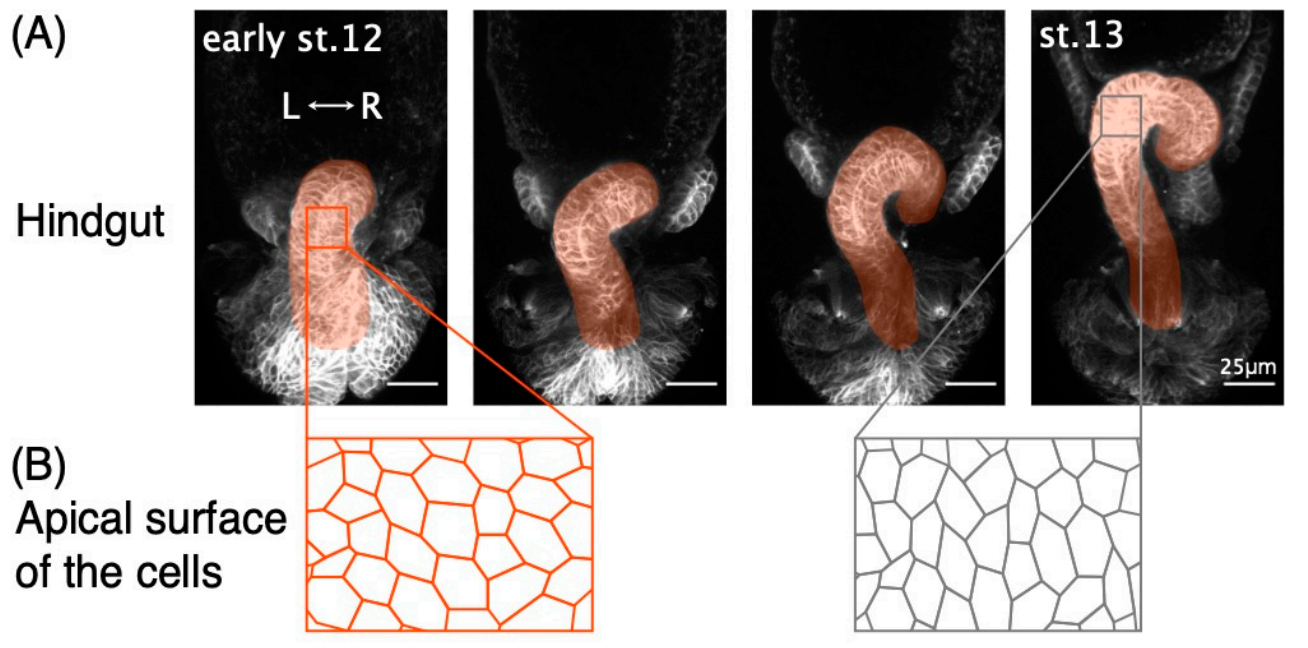

(C)
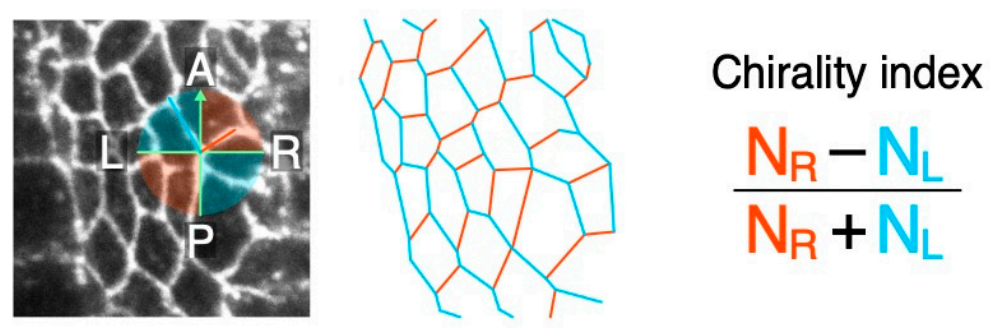

Figure 1. The wild-type Drosophila embryonic hindgut rotates $90^{\circ}$ counterclockwise. (A) The Drosophila embryonic hindgut (orange), visualized by the expression of UAS-myr-GFP (which encodes a cell-membrane marker) driven by byn-Gal4. The hindgut has a bilaterally symmetric structure in which the anterior part curves ventrally at early stage 12. In the wild-type embryo, the hindgut subsequently rotates counterclockwise, causing the organ to curve to the right. At the end of stage 13, the hindgut has a hook shape pointing toward the right. L, left; R, right. (B) Apical cell boundaries in the wild-type hindgut (orange lines) prior to hindgut rotation tend to slant to the left, which is defined as dextral cell chirality. After hindgut rotation, these cells are no longer chiral, but are instead LR-symmetric (gray lines). (C) Schemas that summarize the procedure used to calculate the cell chirality index. The angle between each boundary and the anterior-posterior (AP) axis of the hindgut was determined. LR represents the left-right axis of the hindgut. Each boundary was classified as right-tilted (orange) or left-tilted (turquoise), and the cell chirality index was calculated from the number of right-tilted $\left(N_{R}\right)$ and left-tilted $\left(N_{L}\right)$ boundaries using the formula shown on the right side of the figure. Scale bars in A are $25 \mu \mathrm{m}$. 
We previously showed that the mean cell-chirality index strongly correlates with the percentage of normal hindgut laterality in embryos with various genotypes [29]. To investigate a causal association between the enantiomorphic states of chiral cells and the LR-direction of hindgut rotation, we here analyzed the mathematical characteristics of the correlation between the mean cell-chirality index and the rotational direction of the hindgut. We assigned each embryo a laterality score based on the rotational direction of the hindgut, as 1 (normal LR asymmetry), 0.5 (non-laterality; i.e., bilateral symmetry), or 0 (inverse LR asymmetry) (Figure 2A). We calculated the mean laterality score for all embryos with the same genotype (Figure 2B; the number of embryos is shown as $N_{\mathrm{x}}$ ). Besides the eight genetic conditions analyzed in the previous study, we examined two additional conditions-double mutant embryos of $d a^{10}$ and $e m c^{A P 6}\left(d a^{10} ; e m c^{A P 6}\right)$ and MyoIC overexpressing embryos (NP2432 > MyoIC) - to study the correlation between the mean cell-chirality index and the mean laterality score (Figure 2B) [29]. Our previous analysis revealed that a da mutation suppresses LR defects of the hindgut in the emc mutant because its LR defects are induced through da hyperactivation [29]. We also examined embryos overexpressing UAS-MyoIC, which leads to sinistral LR-asymmetric development in the hindgut epithelium (Figure 2B) [26,40,41].
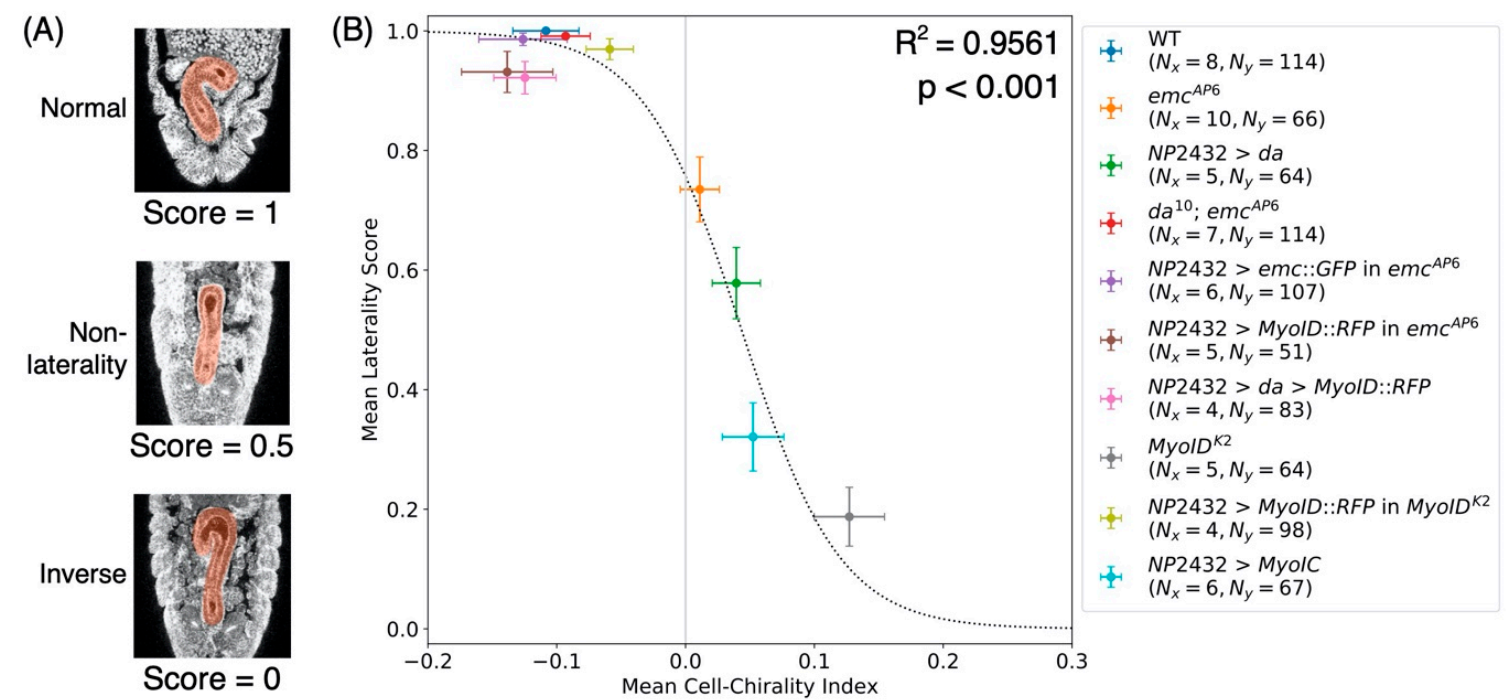

Figure 2. Cell chirality significantly explains the hindgut LR-asymmetry of fixed embryos with various genotypes. (A) Typical examples of the hindgut (shown in orange) demonstrating normal LR asymmetry, non-laterality (bilateral symmetry), and inverse LR asymmetry, which were scored as $1,0.5$, and 0 , respectively. (B) Graph showing the correlation between the mean cell-chirality index and the mean laterality score; colored circles show mean values, and colored lines show standard error. The dotted line indicates the logistic model that best fits the data. Some data were adopted from our previous paper [29]. Genotypes are shown in the right panel. $N_{\mathrm{x}}$ and $N_{\mathrm{y}}$ indicate the number of embryos used to analyze the mean cell-chirality index and the mean laterality score, respectively. $R^{2}$, coefficient of determination; $p<0.001$, two-tailed $t$-test.

We fitted the logistic model (Figure 2B, dotted line) with the cell chirality index as an explanatory variable and the mean laterality score as an objective variable (Figure 2B). The chirality index significantly explained the mean laterality score [quasibinomial generalized linear model (GLM); two-tailed $t$-test, $p<0.001$ ] (Figure 2B; Table A1).

\subsection{The Cell Chirality Index Predicts the LR-Directions of Future Hindgut Rotation in Live Embryos}

Considering that cell chirality appears before hindgut rotation is initiated, the significant correlation found between the mean cell-chirality index and the mean laterality score suggested that an individual embryo's cell chirality index might determine the LR directionality of its hindgut rotation. In this case, the individual's cell chirality index should predict the left or right directionality of the upcoming 
hindgut rotation. To test this possibility, we developed a new procedure to ascertain the cell chirality index in the hindgut of live embryos and to determine the subsequent LR directionality of the hindgut rotation. In this system, the apical cell boundaries of the hindgut epithelium were visualized in live embryos at early stage 12 under a confocal laser microscope. The embryos were cultured for another three hours (when hindgut rotation would be complete in wild-type embryos), at which point we examined the direction of hindgut rotation (Figure 3A, right panel). The live cell-chirality index was calculated for each embryo using the live-imaging data from early stage 12, and the embryo was assigned a laterality score of 1 (normal LR asymmetry), 0.5 (non-laterality), or 0 (inverse LR asymmetry), according to the direction of the subsequent hindgut rotation.

(A)
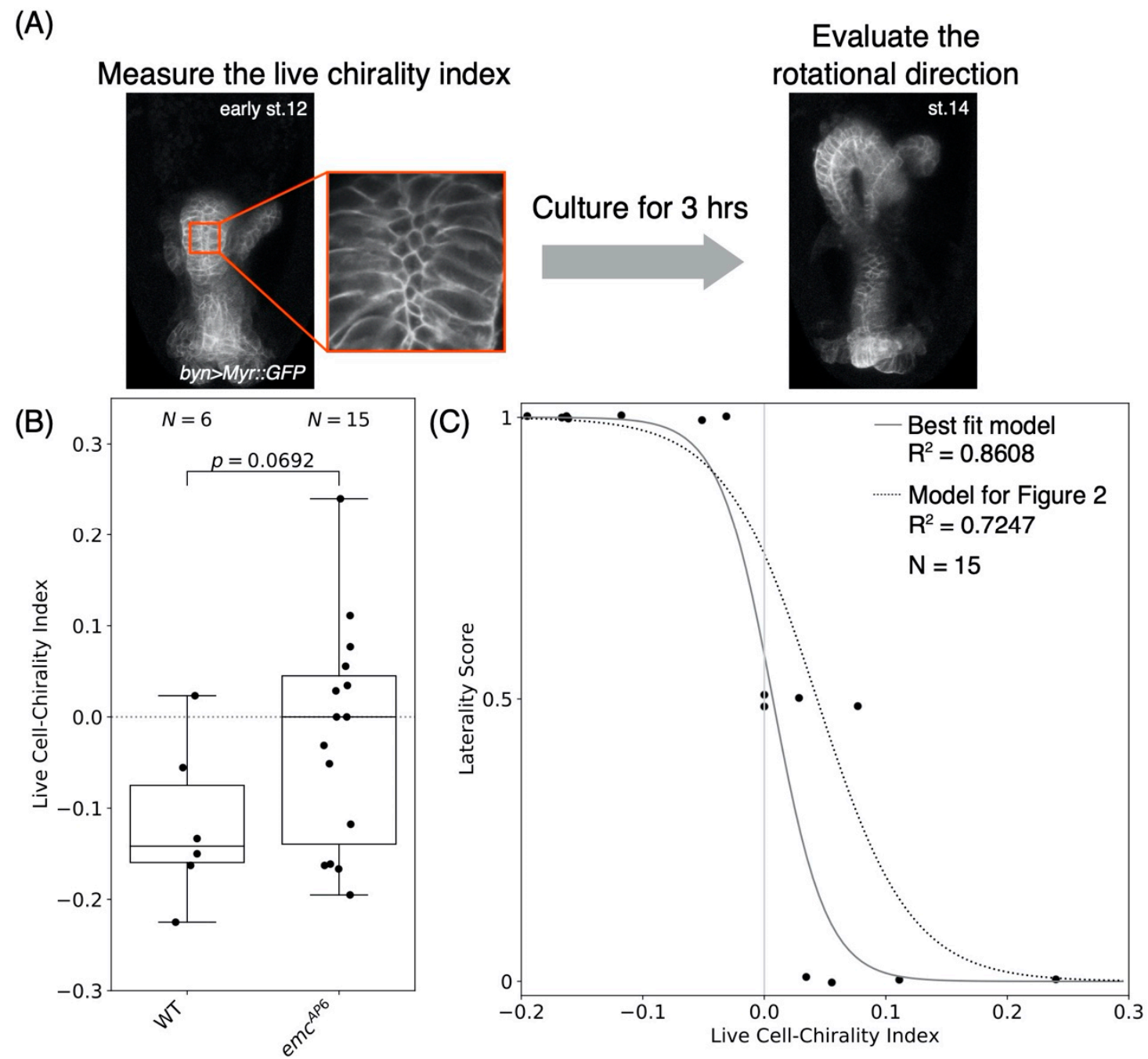

Figure 3. The live cell-chirality index predicts the rotational direction of the hindgut. (A) Typical images of the hindgut in live embryos, showing cell boundaries visualized by UAS-myr::GFP driven by byn-Gal4 in the hindgut epithelium. At early stage 12, the cell-chirality index of the living hindgut epithelial cells was calculated based on a high-magnification image of the region outlined by the orange square (left panel). After imaging, the embryos were incubated for $3 \mathrm{~h}$, at which point each embryo was examined and assigned a laterality score of 1 (normal LR-asymmetry of the hindgut), 0.5 (bilateral symmetry), or 0 (inverse LR-asymmetry). (B) Box plot of the live-cell chirality index of the hindgut in the wild-type and $e m c^{A P 6}$ mutant embryos, showing the number of embryos analyzed $(N)$ and the $p$-value (two-tailed $t$-test). (C) Graph showing the correlation between the live cell-chirality index and the laterality score of the hindgut in the emc mutant. The solid line indicates the best-fitted logistic model (Table A2). The dotted line indicates the logistic model obtained from fixed embryos in Figure 2. $R^{2}$, coefficient of determination; $N$, the number of embryos analyzed. 
To investigate whether the live cell-chirality index correlates with the rotational direction of the hindgut, we used $e m c^{A P 6}$ homozygotes, which have an LR-randomization phenotype. Of the ten different mutants examined in this study, the $e m c^{A P 6}$ mutant was unique in having a mean cell-chirality index of nearly zero with deviations in both the plus and minus values (Figure 2B). In addition, the hindgut in these embryos can develop with normal or inverse LR asymmetry or with a non-lateral phenotype [29]. Thus, we speculated that cell chirality varies stochastically between individual embryos, and that each embryo's predominant cell chirality might induce the hindgut to rotate in the corresponding direction. Based on this assumption, we evaluated the consistency of cell chirality indexes obtained from individual fixed and live $e m c^{A P 6}$ homozygous embryos, and compared the mean cell-chirality index for live wild-type and $e m c^{A P 6}$ homozygous embryos. There was no significant difference in the mean live cell-chirality indexes for wild-type $(-0.12 \pm 0.04)$ and $e m c^{A P 6}$ homozygous embryos $(-0.02 \pm 0.03$; two-tailed $t$-test, $p=0.069217)$, and the values were similar to those reported previously for fixed embryos (wild-type $-0.11 \pm 0.03$; emc ${ }^{A P 6}$ homozygote $0.01 \pm 0.02$ ) [29]. We also found that the live cell-chirality index varied widely for individual emc ${ }^{A P 6}$ homozygotes, with both plus and minus values, unlike that of wild-type embryos (Figure 3B).

Given that the values of the cell-chirality indexes agreed well between live and fixed embryos, we next analyzed statistical correlations between the live cell-chirality index and the laterality score for each $e m c^{A P 6}$-mutant embryo. We fitted the logistic model (solid line, Figure 3B) with the live chirality index as an explanatory variable and the laterality score of each embryo as an objective variable and found that live cell chirality, which was determined prior to the onset of hindgut rotation, significantly explained the LR-directionality of rotation [quasibinomial GLM, two-tailed $t$-test, $p<0.01$ ] (Figure 3C; Table A2). Importantly, the logistic model obtained for fixed embryos (Figures 2B and 3C, dotted line) with the genetic conditions described in Figure 2 significantly fitted the data obtained from each living $e m c^{A P 6}$-mutant embryo [quasibinomial GLM, likelihood ratio test, $\chi_{1}^{2}=4.327, p<0.05$ ], and the model explained $72.5 \%$ of the total variation in the hindgut rotational direction in live $e m c^{A P 6}$-mutant embryos (Table A3). These results suggested that the cell chirality index correlates with the rotational direction of the hindgut, regardless of whether enantiomorphic states of chiral cells are determined by genetics or stochastic fluctuations. Based only on the statistical correlation, we could not conclude whether cell chirality was the cause or effect; however, given the clear chronological sequence of the formation of cell chirality and the initiation of hindgut rotation, we concluded that the enantiomorphic states of chiral cells direct the LR-asymmetry of hindgut rotation.

\section{Discussion}

Previous studies suggest that the enantiomorphic state of chiral cells in the Drosophila hindgut epithelium determines the direction of hindgut rotation, with an average dextral or sinistral cell chirality leading to a counterclockwise or clockwise rotation, respectively $[25,27,29]$. This postulation was first supported by the observation that the mean state of cell chirality correlates to the LR-direction of hindgut rotation in various mutants [25,27,29]. For example, in MyoID mutants, which develop sinistral cell chirality, the hindgut rotates clockwise, whereas wild-type embryos show dextral cell chirality and a counterclockwise rotation [27]. In Drosophila E-cadherin or emc mutants, which have no cell chirality on average, the LR-direction of hindgut rotation is randomized or the hindgut remains LR-symmetric [27,29]. Additional support for this postulation comes from computer vertex-model simulations in which the initial enantiomorphic state of chiral cells introduced into the model defines the LR-direction of the model gut-tube rotation and successively drives tube rotation in the direction determined at the outset [27]. Although studies have consistently reported an apparent concordance between the state of cell chirality and the LR-directionality of hindgut rotation, supported by predictions from computer simulations [25,27], a cause-and-effect relationship has not previously been verified.

To address this issue, in this article we mathematically evaluated this hypothetical causal relationship by analyzing the correlation between the enantiomorphic states of chiral cells and the LR-direction of hindgut rotation. We examined fixed embryos to determine the mean cell-chirality index and laterality 
score for each of ten different genetic conditions, and analyzed the correlation between the cell-chirality index and laterality score. In our logistic model, the mean cell-chirality index, representing each genotype as an explanatory variable, significantly fitted with the mean laterality score (Figure 2B). Therefore, the mechanisms by which cell chirality defines the LR-directionality of hindgut rotation may be similar, regardless of the genetic background. This result also demonstrates that the LR-directionality of hindgut rotation is statistically explained by just the enantiomorphic state of chiral cells as averaged for embryos of the same genotype.

In correlation analysis of fixed embryos, the mean cell-chirality index of $e m c^{A P 6}$-mutant embryos was $0.01 \pm 0.02$; however, all three hindgut-rotation phenotypes-clockwise, counterclockwise, and bilaterally symmetric-were evident among the embryos (Figure 2B) [29]. Thus, one could argue that cell chirality might not be essential for directing hindgut rotation. Our analyses here addressed this question. In this study, we developed a method to determine the live cell-chirality index and the direction of the hindgut rotation in each living embryo. We found that the live cell-chirality indexes representing individual $e m c^{A P 6}$ mutant embryos varied widely, being distributed from plus to minus values, although the mean value of these embryos' live cell-chirality index was nearly zero (Figure 3B). Therefore, this mutant background was particularly useful for analyzing the correlation between the states of cell chirality and the rotational direction of the hindgut in each live embryo. Our logistic model formulated from the data of the live $e m c^{A P 6}$ mutant embryos showed a significant correlation between the live cell-chirality index and the laterality score of individual embryos (Figure 3C). Therefore, in each of these embryos, the live cell-chirality index significantly explained the rotational direction of the hindgut. Based on these results, we ascertained that the cell chirality is not only sufficient, but also required for the hindgut rotation, because our model predicts that living embryos with a zero cell-chirality index tend to have a bilaterally symmetric hindgut.

Importantly, the logistic model obtained from fixed embryos with various genetic conditions significantly fitted the data obtained from each living $e m c^{A P 6}$-mutant embryo [quasibinomial GLM, likelihood ratio test, $\chi_{1}^{2}=4.327, p<0.05$ ], and $72.5 \%$ of the total variation in the rotational direction of the $e m c^{A P 6}$-mutant hindgut could be explained by the model (Figure 3C; Table A3), while 27.5\% of that was explained by residuals. Given the wide range of live cell-chirality indexes in individual embryos from the same $e m c^{A P 6}$-mutant background, cell chirality may be formed stochastically in each individual under this condition. Nevertheless, the logistic model formulated from the data from fixed embryos of various genotypes significantly explains the data that presumably reflect stochastic events in each $e m c^{A P 6}$ individual. Therefore, the underlying mechanisms by which cell chirality drives the LR-directional hindgut rotation are common between genetically or stochastically formed cell chirality.

In this study, we mathematically confirmed the causal relationship between cell chirality and the direction of hindgut rotation. Our logistic model also implies that cell chirality is sufficient and necessary for the hindgut rotation. Considering the time course of the events, in which cell chirality is formed before the initiation of the hindgut rotation in vivo, our results presented here demonstrate that cell chirality is the primary cause of the hindgut rotation. Since cell chirality is involved in the LR-asymmetric development of various organs in Drosophila, such a cause-and-effect relationship can also be applied to explain their morphogenesis. Furthermore, our idea could extend to LR-asymmetric development involving chiral cells in other species.

Author Contributions: Conceptualization-T.I. and K.M.; Methodology-T.I.; Investigation-T.I. and M.I.; Data Analysis-T.I.; Writing-Original Draft Preparation-T.I.; Writing—Review \& Editing-T.I., M.I., and K.M.; Supervision-K.M.; Funding Acquisition-T.I., M.I., and K.M. All authors have read and agreed to the published version of the manuscript.

Funding: This study was supported by Japan Society for the Promotion of Science KAKENHI Grants (\#16J01027 to T.I., \#18K06255 to M.I., and \#15H05856 and \#15H05863 to K.M.)

Acknowledgments: We thank N.S. Moon and S. Noselli for fly stocks. We also thank the Bloomington Drosophila Stock Center (Indiana University), the Drosophila Genetic Resource Center (Indiana University), and the Kyoto 
Stock Center (Kyoto Institute of Technology) for fly stocks, and the Developmental Studies Hybridoma Bank (University of Iowa) for antibodies.

Conflicts of Interest: The authors declare no conflict of interest.

\section{Appendix A}

Table A1. Factors affecting variations in the laterality score of the embryonic hindgut in various Drosophila genotypes.

\begin{tabular}{ccc}
\hline & $t_{8}$-Value & $p$-Value \\
\hline Intercept & -4.042 & 0.003723 \\
Chirality Index & -5.992 & 0.000326 \\
\hline
\end{tabular}

Table A2. Factors affecting variations in the rotational direction of the hindgut in $e m c^{A P 6}$ mutants.

\begin{tabular}{ccc}
\hline & $\boldsymbol{t}_{13}$-Value & $\boldsymbol{p}$-Value \\
\hline Intercept & -1.149 & 0.27109 \\
Live Chirality Index & -3.256 & 0.00625 \\
\hline
\end{tabular}

Table A3. Logistic regression analyses between the laterality score and live cell-chirality index in emc ${ }^{A P 6}$ mutants.

\begin{tabular}{ccc}
\hline & Model & $\boldsymbol{R}^{2}$ \\
\hline Best fit model & $1-\frac{1}{1+e^{-4.531 \times(x-0.00697)}}$ & 0.8607872 \\
Model fitted for Figure 2 & $1-\frac{1}{1+e^{-0.410 \times(x-3.42)}}$ & 0.7246853 \\
\hline
\end{tabular}

\section{References}

1. Blum:, M.; Ott, T. Animal left-right asymmetry. Curr. Biol. 2018, 28, R301-R304. [CrossRef]

2. Hirokawa, N.; Tanaka, Y.; Okada, Y.; Takeda, S. Nodal Flow and the Generation of Left-Right Asymmetry. Cell 2006, 125, 33-45. [CrossRef]

3. Nakamura, T.; Hamada, H. Left-right patterning: Conserved and divergent mechanisms. Development 2012, 139, 3257-3262. [CrossRef] [PubMed]

4. Levin, M. Left-right asymmetry in embryonic development: A comprehensive review. Mech. Dev. 2005, 122, 3-25. [CrossRef] [PubMed]

5. Yoshiba, S.; Hamada, H. Roles of cilia, fluid flow, and Ca2+signaling in breaking of left-right symmetry. Trends Genet. 2014, 30, 10-17. [CrossRef] [PubMed]

6. Nonaka, S.; Tanaka, Y.; Okada, Y.; Takeda, S.; Harada, A.; Kanai, Y.; Kido, M.; Hirokawa, N. Randomization of left-right asymmetry due to loss of nodal cilia generating leftward flow of extraembryonic fluid in mice lacking KIF3B motor protein. Cell 1998, 95, 829-837. [CrossRef]

7. Nonaka, S.; Shiratori, H.; Saijoh, Y.; Hamada, H. Determination of left-right patterning of the mouse embryo by artificial nodal flow. Nature 2002, 418, 96-99. [CrossRef]

8. Takeda, S.; Yonekawa, Y.; Tanaka, Y.; Okada, Y.; Nonaka, S.; Hirokawa, N. Left-right asymmetry and kinesin superfamily protein KIF3a: New insights in determination of laterality and mesoderm induction by KIF3A(-/-) mice analysis. J. Cell Biol. 1999, 145, 825-836. [CrossRef]

9. Kajikawa, E.; Horo, U.; Ide, T.; Mizuno, K.; Minegishi, K.; Hara, Y.; Ikawa, Y.; Nishimura, H.; Uchikawa, M.; Kiyonari, H.; et al. Nodal paralogues underlie distinct mechanisms for visceral left-right asymmetry in reptiles and mammals. Nat. Ecol. Evol. 2020, 4, 261-269. [CrossRef]

10. Tabin, C. Do we know anything about how left-right asymmetry is first established in the vertebrate embryo? J. Mol. Histol. 2005, 36, 317-323. [CrossRef]

11. Inaki, M.; Liu, J.; Matsuno, K. Cell chirality: Its origin and roles in left-right asymmetric development. Philos. Trans. R. Soc. B Biol. Sci. 2016, 371, 20150403. [CrossRef] 
12. Inaki, M.; Sasamura, T.; Matsuno, K. Cell chirality drives left-right asymmetric morphogenesis. Front. Cell Dev. Biol. 2018, 6. [CrossRef]

13. Utsunomiya, S.; Sakamura, S.; Sasamura, T.; Ishibashi, T.; Maeda, C.; Inaki, M.; Matsuno, K. Cells with Broken Left-Right Symmetry: Roles of Intrinsic Cell Chirality in Left-Right Asymmetric Epithelial Morphogenesis. Symmetry 2019, 11, 505. [CrossRef]

14. Kuroda, R.; Endo, B.; Abe, M.; Shimizu, M. Chiral blastomere arrangement dictates zygotic left-right asymmetry pathway in snails. Nature 2009, 462, 790-794. [CrossRef]

15. Naganathan, S.R.; Fürthauer, S.; Nishikawa, M.; Jülicher, F.; Grill, S.W. Active torque generation by the actomyosin cell cortex drives left-right symmetry breaking. Elife 2014, 3, e04165. [CrossRef]

16. Hayashi, T.; Murakami, R. Left-right asymmetry in Drosophila melanogaster gut development. Dev. Growth Differ. 2001, 43, 239-246. [CrossRef]

17. Hozumi, S.; Maeda, R.; Taniguchi, K.; Kanai, M.; Shirakabe, S.; Sasamura, T.; Spéder, P.; Noselli, S.; Aigaki, T.; Murakami, R.; et al. An unconventional myosin in Drosophila reverses the default handedness in visceral organs. Nature 2006, 440, 798-802. [CrossRef]

18. Ligoxygakis, P.; Strigini, M.; Averof, M. Specification of left-right asymmetry in the embryonic gut of Drosophila. Development 2001, 128, 1171-1174.

19. Pascual, A.; Huang, K.L.; Neveu, J.; Préat, T. Brain asymmetry and long-term memory: Fruitflies that have structurally similar brain hemispheres forget within a matter of hours. Nature 2004, 427, 605-606. [CrossRef]

20. Spéder, P.; Ádám, G.; Noselli, S. Type ID unconventional myosin controls left-right asymmetry in Drosophila. Nature 2006, 440, 803-807. [CrossRef]

21. Maeda, R.; Hozumi, S.; Taniguchi, K.; Sasamura, T.; Murakami, R.; Matsuno, K. Roles of single-minded in the left-right asymmetric development of the Drosophila embryonic gut. Mech. Dev. 2007, 124, 204-217. [CrossRef]

22. Nakamura, M.; Matsumoto, K.; Iwamoto, Y.; Muguruma, T.; Nakazawa, N.; Hatori, R.; Taniguchi, K.; Maeda, R.; Matsuno, K. Reduced cell number in the hindgut epithelium disrupts hindgut left-right asymmetry in a mutant of pebble, encoding a RhoGEF, in Drosophila embryos. Mech. Dev. 2013, 130, 169-180. [CrossRef]

23. Taniguchi, K.; Hozumi, S.; Maeda, R.; Ooike, M.; Sasamura, T.; Aigaki, T.; Matsuno, K. D-JNK signaling in visceral muscle cells controls the laterality of the Drosophila gut. Dev. Biol. 2007, 311, 251-263. [CrossRef]

24. Kuroda, J.; Nakamura, M.; Yoshida, M.; Yamamoto, H.; Maeda, T.; Taniguchi, K.; Nakazawa, N.; Hatori, R.; Ishio, A.; Ozaki, A.; et al. Canonical Wnt signaling in the visceral muscle is required for left-right asymmetric development of the Drosophila midgut. Mech. Dev. 2012, 128, 625-639. [CrossRef]

25. Inaki, M.; Hatori, R.; Nakazawa, N.; Okumura, T.; Ishibashi, T.; Kikuta, J.; Ishii, M.; Matsuno, K.; Honda, H. Chiral cell sliding drives left-right asymmetric organ twisting. Elife 2018, 7, e32506. [CrossRef]

26. Hozumi, S.; Maeda, R.; Taniguchi-Kanai, M.; Okumura, T.; Taniguchi, K.; Kawakatsu, Y.; Nakazawa, N.; Hatori, R.; Matsuno, K. Head region of unconventional myosin I family members is responsible for the organ-specificity of their roles in left-right polarity in Drosophila. Dev. Dyn. 2008, 237, 3528-3537. [CrossRef]

27. Taniguchi, K.; Maeda, R.; Ando, T.; Okumura, T.; Nakazawa, N.; Hatori, R.; Nakamura, M.; Hozumi, S.; Fujiwara, H.; Matsuno, K. Chirality in planar cell shape contributes to left-right asymmetric epithelial morphogenesis. Science 2011, 333, 339-341. [CrossRef]

28. Hatori, R.; Ando, T.; Sasamura, T.; Nakazawa, N.; Nakamura, M.; Taniguchi, K.; Hozumi, S.; Kikuta, J.; Ishii, M.; Matsuno, K. Left-right asymmetry is formed in individual cells by intrinsic cell chirality. Mech. Dev. 2014, 133, 146-162. [CrossRef]

29. Ishibashi, T.; Hatori, R.; Maeda, R.; Nakamura, M.; Taguchi, T.; Matsuyama, Y.; Matsuno, K. E and ID proteins regulate cell chirality and left-right asymmetric development in Drosophila. Genes Cells 2019, 24, 214-230. [CrossRef]

30. Sato, K.; Hiraiwa, T.; Maekawa, E.; Isomura, A.; Shibata, T.; Kuranaga, E. Left-right asymmetric cell intercalation drives directional collective cell movement in epithelial morphogenesis. Nat. Commun. 2015, 6. [CrossRef]

31. Coutelis, J.B.; Géminard, C.; Spéder, P.; Suzanne, M.; Petzoldt, A.G.; Noselli, S. Drosophila Left/Right Asymmetry Establishment Is Controlled by the Hox Gene Abdominal-B. Dev. Cell 2013, 24, 89-97. [CrossRef] [PubMed] 
32. González-Morales, N.; Géminard, C.; Lebreton, G.; Cerezo, D.; Coutelis, J.B.; Noselli, S. The Atypical Cadherin Dachsous Controls Left-Right Asymmetry in Drosophila. Dev. Cell 2015, 33, 675-689. [CrossRef]

33. Ray, P.; Chin, A.S.; Worley, K.E.; Fan, J.; Kaur, G.; Wu, M.; Wan, L.Q. Intrinsic cellular chirality regulates left-right symmetry breaking during cardiac looping. Proc. Natl. Acad. Sci. USA 2018, 115, E11568-E11577. [CrossRef]

34. Onuma, T.A.; Hayashi, M.; Gyoja, F.; Kishi, K.; Wang, K.; Nishida, H. A chordate species lacking Nodal utilizes calcium oscillation and Bmp for left-right patterning. Proc. Natl. Acad. Sci. USA 2020, 117, 4188-4198. [CrossRef]

35. Chen, T.H.; Hsu, J.J.; Zhao, X.; Guo, C.; Wong, M.N.; Huang, Y.; Li, Z.; Garfinkel, A.; Ho, C.M.; Tintut, Y.; et al. Left-right symmetry breaking in tissue morphogenesis via cytoskeletal mechanics. Circ. Res. 2012, 110, 551-559. [CrossRef]

36. Tamada, A.; Kawase, S.; Murakami, F.; Kamiguchi, H. Autonomous right-screw rotation of growth cone filopodia drives neurite turning. J. Cell Biol. 2010, 188, 429-441. [CrossRef]

37. Tee, Y.H.; Shemesh, T.; Thiagarajan, V.; Hariadi, R.F.; Anderson, K.L.; Page, C.; Volkmann, N.; Hanein, D.; Sivaramakrishnan, S.; Kozlov, M.M.; et al. Cellular chirality arising from the self-organization of the actin cytoskeleton. Nat. Cell Biol. 2015, 17, 445-457. [CrossRef]

38. Wan, L.Q.; Ronaldson, K.; Park, M.; Taylor, G.; Zhang, Y.; Gimble, J.M.; Vunjak-Novakovic, G. Micropatterned mammalian cells exhibit phenotype-specific left-right asymmetry. Proc. Natl. Acad. Sci. USA 2011, 108, 12295-12300. [CrossRef]

39. Xu, J.; Van Keymeulen, A.; Wakida, N.M.; Carlton, P.; Berns, M.W.; Bourne, H.R. Polarity reveals intrinsic cell chirality. Proc. Natl. Acad. Sci. USA 2007, 104, 9296-9300. [CrossRef]

40. Lebreton, G.; Géminard, C.; Lapraz, F.; Pyrpassopoulos, S.; Cerezo, D.; Spéder, P.; Ostap, E.M.; Noselli, S. Molecular to organismal chirality is induced by the conserved myosin 1D. Science 2018, 362, 949-952. [CrossRef]

41. Okumura, T.; Sasamura, T.; Inatomi, M.; Hozumi, S.; Nakamura, M.; Hatori, R.; Taniguchi, K.; Nakazawa, N.; Suzuki, E.; Maeda, R.; et al. Class I myosins have overlapping and specialized functions in left-right asymmetric development in Drosophila. Genetics 2015, 199, 1183-1199. [CrossRef]

42. Wang, L.H.; Baker, N.E. E Proteins and ID Proteins: Helix-Loop-Helix Partners in Development and Disease. Dev. Cell 2015, 35, 269-280. [CrossRef]

43. Ellis, H.M. Embryonic expression and function of the Drosophila helix-loop-helix gene, extramacrochaetae. Mech. Dev. 1994, 47, 65-72. [CrossRef]

44. Wülbeck, C.; Fromental-Ramain, C.; Campos-Ortega, J.A. The HLH domain of a zebrafish hE12 homologue can partially substitute for functions of the HLH domain of Drosophila daughterless. Mech. Dev. 1994, 46, 73-85. [CrossRef]

45. Petzoldt, A.G.; Coutelis, J.-B.; Géminard, C.; Spéder, P.; Suzanne, M.; Cerezo, D.; Noselli, S. DE-Cadherin regulates unconventional Myosin ID and Myosin IC in Drosophila left-right asymmetry establishment. Development 2012, 139, 1874-1884. [CrossRef]

46. Popova, M.K.; He, W.; Korenjak, M.; Dyson, N.J.; Moon, N.-S. Rb deficiency during Drosophila eye development deregulates EMC, causing defects in the development of photoreceptors and cone cells. J. Cell Sci. 2011, 124, 4203-4212. [CrossRef]

47. Giebel, B.; Stüttem, I.; Hinz, U.; Campos-Ortega, J.A. Lethal of Scute requires overexpression of Daughterless to elicit ectopic neuronal development during embryogenesis in Drosophila. Mech. Dev. 1997, 63, 75-87. [CrossRef]

48. Pfeiffer, B.D.; Truman, J.W.; Rubin, G.M. Using translational enhancers to increase transgene expression in Drosophila. Proc. Natl. Acad. Sci. USA 2012, 109, 6626-6631. [CrossRef]

49. Hayashi, S.; Ito, K.; Sado, Y.; Taniguchi, M.; Akimoto, A.; Takeuchi, H.; Aigaki, T.; Matsuzaki, F.; Nakagoshi, H.; Tanimura, T.; et al. GETDB, a database compiling expression patterns and molecular locations of a collection of gal4 enhancer traps. Genesis 2002, 34, 58-61. [CrossRef]

50. Brand, A.H.; Perrimon, N. Targeted gene expression as a means of altering cell fates and generating dominant phenotypes. Development 1993, 118, 401-415.

51. Schindelin, J.; Arganda-Carreras, I.; Frise, E.; Kaynig, V.; Longair, M.; Pietzsch, T.; Preibisch, S.; Rueden, C.; Saalfeld, S.; Schmid, B.; et al. Fiji: An open-source platform for biological-image analysis. Nat. Methods 2012, 9, 676-682. [CrossRef] [PubMed] 
52. Campbell, M.J.; Dobson, A.J. An Introduction to Generalized Linear Models. Biometrics 1991, $47,347$. [CrossRef]

53. R Core Team. R: A Language and Environment for Statistical Computing; R Foundation for Statistical Computing: Vienna, Austria, 2020.

54. Van Rossum, G.; Drake, F.L. Python Tutorial, Technical Report CS-R9526. In Proceedings of the Centrum Voor Wiskunde en Informatica (CWI); Academic Press: Cambridge, MA, USA, 1995.

55. Hunter, J.D. Matplotlib: A 2D graphics environment. Comput. Sci. Eng. 2007, 9, 99-104. [CrossRef]

Publisher's Note: MDPI stays neutral with regard to jurisdictional claims in published maps and institutional affiliations.

(C) 2020 by the authors. Licensee MDPI, Basel, Switzerland. This article is an open access article distributed under the terms and conditions of the Creative Commons Attribution (CC BY) license (http://creativecommons.org/licenses/by/4.0/). 